Military Technical College, Kobry El-Kobbah, Cairo, Egypt $9^{\text {th }}$ International Conference

On Aerospace Sciences \& Aviation Technology

\title{
IMPROVEMENT OF RECEIVER OPERATING CHARACTERISTICS USING FAST COMPUTED SPECTRAL CORRELATION FUNCTION
}

\author{
K. El-barbary", E. E. Azzouz", A. Al-Makhlouf"
}

\begin{abstract}
This paper is concerned with the improvement of the receiver operating characteristics. For this purpose, the received signal is interpreted as a cyclostationary process whose statistics vary periodically with time. Three types of detectors are considered. These detectors are the radiometer, the bit-rate single cycle detector, and doubled-carrier single cycle detector. It is found that, the performance of the bit-rate single cycle detector is better than the radiometer. Furthermore, the doubled-carrier single cycle detector is superior from the point of view of the obtained receiver operating characteristic. This result is referred to the fact that the accompanied additive white Gaussian noise (AWGN) to the desired signal is stationary process and it does not have cyclic spectrum component at any cyclic frequency. Results indicate that, at $\mathrm{S} \quad 5 \mathrm{~dB}$, for a probability of false alarm of $2 \%$, the radiometer has a probability of detection of $40 \%$, the bit-rate single cycle detector has a probability of detection of $70 \%$, and the doubled-carrier single cycle detector has a probability of detection of $93 \%$.
\end{abstract}

\section{KEYWORDS}

Cyclostationary, spectral correlation function, and receiver operating characteristics.

\footnotetext{
- Egyption Armed Forces

Syrian Armed Forces
} 


\section{1- INTRODUCTION}

Interception of communication signals is attempted for a variety of reasons including reconnaissance, surveillance and radar, position fixing, identification, and communications jamming. For example, an aircraft might attempt to intercept the communications between a submarine or ship and a satellite, or a satellite might attempt to intercept ground-to-ground communications. Typically, the interceptor has knowledge of no more than the communicator frequency band and modulation format. In the past, most appropriate approaches for signal interception were based on radiometry [1]. However, it is commonly recognized that such radiometric methods can be highly susceptible to unknown and changing noise levels and interference activity. There have been many proposals for counteracting such complications, including various approaches to adjust or adapt threshold levels, adaptive filtering, and directional nulling of interfering signals. But these problems remain as the most serious impediment to signal interception tasks [1].

The radiometric approach for interception is based on the use of stationary random processes as models for signals to be intercepted. However, it is found that, for the purposes of signal interception, the signal of interest is more appropriately modeled as a cyclostationary random process. That is, random process whos statistical parameters vary periodically with time [2]. The message contained in the modulated signal is unknown, and is usually modeled as a stationary random process. This stationarity coupled with the periodicity of sine wave carrier, pulse trains, repeating yclostationary model for the modulated signal.

These cyclostationary signals typically do not exhibit spectral lines because the spectral lines of the unmodulated carrier and/or pulse trains are spread out over relatively broad bands by the stationary random modulation.

Before proceeding to the technical part of this paper, let us consider the signal interception problem and the weaknesses and strengths of radiometry and cyclicfeature detection in a little more detail. There is a clear trend towards increased use of systems employing sophisticated signal formats such as direct-sequence and frequency-hopped spread spectrum modulation. These techniques are to aid communication in the jamming environment and to protect the communication system against interception. In these applications, cyclostationarity performs required signal interception or subsequent analysis tasks unlike the conventional interceptor. In addition, the modulation format of the signal of interest can make it indistinguishable from the background noise. The presence of several identically distributed spectrally superimposed signals will confuse most energy interception schemes, preventing the interceptor from determining any more than knowledge that signals are present in the environment. In particular, energy detection schemes are inherently unable to measure or exploit timing or phasing properties (carrier frequency, chip, or baud timing) of the signals of interest or interferences because these energy detectors usually cannot exploit the cyclostationarity of the signal characteristics.

There are some important advantages of cyclic spectral analysis over energy detection techniques [1]. One of them is its discriminatory capability. Signal features are discretely distributed in cyclic spectrum, even if the signal has continuous 
distribution in the power spectrum. Thus, signals with overlapping featurs in the power spectrum can have non-overlapping features in the cyclic spectrum. Background noise, for instance, has no features at nonzero cyclic frequencies; i.e. analyzing the cyclic spectrum at a nonzero cyclic frequency where a signal-of-interest is expected to appear without any component due to the background noise. Another advantage of cyclic spectral analysis is that the cyclic spectrum is a richer domain for signal analysis than the conventional power spectrum. In addition to the signal separation provided by the cyclic spectrum magnitude, sine-wave carrier, pulse-train frequency, and phase parameters can also be measured from the cyclic spectrum magnitude and phase.

In section 2, theoretical background and basic terminologies for spectral correlation function are presented. In section 3, cyclic-feature detection is discussed and new types of receivers are provided for detection instead of the radiometer. In section 4 , receiver operating characteristics are evaluated through computers simulations, where improvement on the ROC by cyclic detector is indicated. Finally, conclusions of this work are introduced in section 5 .

\section{2- THEORETICAL BACKGROUND AND BASIC TERMINOLOGIES}

Cyclostationarity is a physical phenomenor that describes a probabilistic model for certain random data that involve certain periodicity model. For example, in mechanical-vibration monitoring and diagnosis for machinery, periodicity arises from rotation, revolution, and reciprocation of gears, belts, chains, shafts, pistons, and so on. Also, in communications telemetry, radar, and sonar, periodicity arises from sampling, scanning, modulation, multiplexing, and coding operations. For these and many other examples, the periodicity is an important characteristic that should be reflected in an appropriate probabilistic model.

A process, $x(t)$, is said to be cyclostationary in a wide sense if its mean and autocorrelation are periodic with some period, $T$; i.e.

$$
m_{x}(t+T)=m_{x}(t)
$$

and

$$
R_{x}(t+T, u+T)=R_{x}(t, u),
$$

for all $t$ and $u$ where $m_{x}(t)=E\{x(t)\}$ and $R_{x}(t, u)=E\left\{x(t) x^{*}(u)\right\}$. We shall focus our attention on the autocorrelation function. Since equation (2) can be re-expressed as

$$
R_{x}\left(t+T+\frac{\tau}{2}, t+T-\frac{\tau}{2}\right)=R_{x}\left(t+\frac{\tau}{2}, t-\frac{\tau}{2}\right),
$$

where $t$ and are independent variables. Thus, $R_{x}\left(t+\frac{\tau}{2}, t-\frac{\tau}{2}\right)$, is a periodic in $t$ with period $T \neq 0$ for each value of , and it is defined by

$$
R_{x}\left(t+\frac{\tau}{2}, t-\frac{\tau}{2}\right) \stackrel{\Delta}{=} E\left\{x\left(t+\frac{\tau}{2}\right) x \cdot\left(t-\frac{\tau}{2}\right)\right\},
$$


It is assumed that the Fourier series representation for this periodic function converges. Thus, $R_{x}$ can be expressed as :

$$
R_{x}\left(t+\frac{\tau}{2}, t-\frac{\tau}{2}\right)=\sum_{\alpha} R_{x}^{\alpha}(\tau) e^{j 2 \pi \alpha t},
$$

for which $\left\{R_{x}^{\alpha}(\tau)\right\}$ are the Fourier coefficients, and they are given by

$$
R_{x}^{\alpha}(\tau) \stackrel{\Delta}{=} \frac{1}{T} \int_{-T / 2}^{T / 2} R_{x}\left(t+\frac{\tau}{2}, t-\frac{\tau}{2}\right) e^{-j 2 \pi \alpha t} d t,
$$

and $\alpha$ ranges over all integer multiples of the parameter of periodicity $(1 / T)$ (such as carrier frequency, baud rate, chip rate, hop rate, and their sums and differences). This model for the autocorrelation function $R_{x}$ is adequate for a phenomenon with a single periodicity. However, for a phenomenon with more than one periodicity it must be generalized. This is easily accomplished by letting $\alpha$ in equation (5) range over all integer multiples of all parameters of periodicity of interest, say $1 / T_{1}, 1 / T_{2}, 1 / T_{3} \quad$ e equation (6) is modified as follows :

$$
R_{x}^{\alpha}(\tau) \stackrel{\Delta}{=} \lim _{Z \rightarrow \infty} \frac{1}{Z} \int_{-Z / 2}^{Z / 2} R_{x}\left(t+\frac{\tau}{2}, t-\frac{\tau}{2}\right) e^{-j 2 \pi \alpha t} d t .
$$

where $Z$ is the common multiple of all parameters of periodicity. Such a process is said to be almost cyclostationary in the wide sense, by analogy with the fact that the function $R_{x}\left(t+\frac{\tau}{2}, t-\frac{\tau}{2}\right)$ is said to be an almost periodic function of $t$ [2]. More generally, a non-stationary process $x(t)$ is said to exhibit cyclostationarity if there exists a periodicity parameter $\alpha$ for which the Fourier coefficient defined by equation (7) is not identically zero. The set $\left\{\alpha: R_{x}^{\alpha}(\tau) \neq 0\right\}$ is referred to the set of cyclic frequencies. If $x(t)$ is a cycloergodic process $[2,4]$, which will be the case if an appropriate model is used, then after substitution of (4) into (7), the expectation operator can be omitted to obtain

$$
\hat{R}_{x}^{\alpha}(\tau)=\lim _{z \rightarrow \infty} \frac{1}{z} \int_{-z / 2}^{z / 2} x(t+\tau / 2) x(t-\tau / 2) e^{-i 2 \pi \alpha t} d t .
$$

By analogy with the terminology for conventional autocorrelation $(\alpha=0$ in (5)), the Fourier transform of the cyclic autocorrelation, $S_{x}^{\alpha}(f)$, is given by,

$$
S_{x}^{\alpha}(f) \stackrel{\Delta}{\Delta} \int_{-\infty}^{+\infty} R_{x}^{\alpha}(\tau) e^{-i 2 \pi f \tau} d \tau,
$$

and it is called the spectral correlation function. It can be shown that (8) is expressed by

$$
R_{x}^{\alpha}(\tau)=\lim _{\Delta t \rightarrow \infty} \frac{1}{\Delta t} \int_{-\Delta t / 2}^{\Delta t / 2} u^{\alpha}(t+\tau / 2) v^{\alpha^{*}}(t-\tau / 2) d t
$$

where

$$
u^{\alpha}(t)=x(t) e^{-i \pi \alpha t}, \quad \text { and } \quad v^{\alpha}(t)=x(t) e^{+i \pi \alpha t}
$$


Thus, $R_{x}^{\alpha}(\tau)$ is the cross-correlation between $u^{\alpha}(t)$ and $v^{\alpha}(t)$, and $S_{x}^{\alpha}(f)$ is the Fourier transform of (10) as defined by (9).

A noval method to compute the spectral correlation function was presented in [6]. This method was called frequency-domain method and it comprises the following steps:

1- Two frequency shifted version from the original signal $x(t)$ are formed such as

$x_{1}^{\alpha}(t)=x(t) e^{j \pi \alpha t} \quad$ and

$x_{2}^{\alpha}(t)=x(t) e^{-j \pi \alpha t}$.

2- Instead of computing the time-domain cross-correlation between $x_{1}^{\alpha}(t)$ and $x_{2}^{\alpha}(t)$, then the Fourier transform to find $S_{x}^{\alpha}(f)$, the Fourier transform is applied directly to $x_{1}^{\alpha}(t)$ and $x_{2}^{\alpha}(t)$ as follows

$X_{1}^{\alpha}(f)=F . T .\left\{x_{1}^{\alpha}(t)\right\}$ and

$X_{2}^{\alpha}(f)=F . T .\left\{x_{2}^{\alpha}(t)\right\}$.

3- The spectral correlation function $S_{x}^{\alpha}(f)$ is computed as the average of the product $X_{1}^{\alpha}(f), X_{2}^{\alpha}(f)$, that,

$$
S_{x}^{\alpha}(f)=E\left\{X_{1}^{\alpha}(f) \operatorname{conj}\left(X_{2}^{\alpha}(f)\right)\right\}
$$

It is found, in [6], that, the computation time of the spectral correlation function using the time-domain (T-D) method, equations (8) and (9), is much longer than the computation time needed using the proposed frequency-domain (F-D) method, equations (12)-(14), for any selected test signal. Additionally, the cyclic spectra resulted by using the frequency-domain method is more smoothing than that resulted by using the time-domain method. Fig. 1, shows the spectral correlation function of BPSK and QPSK signals using time-domain method and frequency-domain method.

\section{3- CYCLIC DETECTION}

For the purposes of signal interception, If there is more than one source of periodicity and the periods are not all commensurable, then the process is called almost cyclostationary since their parameters are almost periodic functions of time.

The detection problem considered here can be stated in terms of a binary hypothesis test, with the null hypothesis $H_{0}$ corresponding to signal absent

$$
H_{0}: x(t)=n(t)
$$

and the alternative hypothesis $H_{1}$ corresponding to signal present

$H_{1}: x(t)=n(t)+s(t)$

during the sliding observation interval centered at $t$ with length $T$. The function $x(t)$ represents the received data, $n(t)$ is white Gaussian noise (WGN), and $s(t)$ is the zero-mean random signal of interest (SOI), which is assumed to be weak and independent of the noise. Further, the signal of interest is most appropriately 
detector output $y_{s c}(t)$ is a measure of the amount of spectral correlation present in the received waveform, whereas the radiometer output $y_{r}(t)$ is a measure of the amount of energy present in the received waveform.

\section{4-COMPUTER SIMULATION FOR THE RECEIVER OPERATING CHARACTERRISTICS}

Consider the following two hypotheses

$$
\begin{aligned}
& H_{0}: x(t)=n(t), \\
& H_{1}: x(t)=n(t)+s(t),
\end{aligned}
$$

where $x(t)$ is the received signal, $s(t)$ is the signal of interest, and $n(t)$ is the noise sequence. Under these two hypotheses the receive-operating characteristics, ROC, could be obtained. The ROC determines the maximum achieved probability of detection $P_{D}$ for certain level of probability falo alarm $P_{F A}$. The probability of detection $P_{D}$ is the probability that $x(t)$ eveeds the threshold when $H_{1}$ is true while the probability faise alarm $P_{F A}$ is the ubability that $x(t)$ exceeds the threshold when $H_{0}$ is true. Detectro under $s^{t}, y$ are the radiometry (19) and the single cycle detators for bit-rate ox doubled carrier frequency $\alpha=r_{b}, \alpha=2 f_{c}$ in (20) resper. efly. In order to obtain $P_{D}$ and $P_{F A}$, it is necessary to generate many ernple of the signal and noise processes in order to obtain adequate sample sets of detection statistics. The generated statistics are compared to thresholds to estimate the required probabilities.

Receiver operating characteristics are evaluated for both BPSK and the QPSK signals of interest. For each type of signals of interest, two lengths are considered. The first length is of 128 samples (16 symbois) per a segment and the second length is of 256 samples ( 32 symbols) per a segment. To achieve the value of the permissible probability of false alarm, a sequence of 100 segments of noise only is used for each case. Each one of the aforementioned cases is discussed at two values of signal to noise ratio which are $\mathrm{SNR}=-5 \mathrm{~dB}$ and $\mathrm{SNR}=-10 \mathrm{~dB}$. For all cases, the bit rate is $r_{b}=100 \mathrm{KHz}$, the carrier frequency is $f_{c}=200 \mathrm{KHz}$, and the sampling rate is $f_{s}=800 \mathrm{KHz}$. All results of these considered cases are illustrated at Figs. 2-9. In all figures, the ROC of the radiometer is presented by a line with stars, the ROC of the bit-rate singie cycle detector is presented by a line with small circles, and the ROC of the doubled-carrier single cycle detector is presented by a solid line. For cases of the BPSK signal of interest, there are R

radiometer, the bit-rate single cycle detector, and the doubled-carrier single cycle detector. The results are presented in Figs. 2, 3, 6, and 7. Meanwhile for the QPSK the radiometer detector, and the bit-rate single cycle detector. The results are presented in Figs. 4, 5, 8, and 9 . That is, the ROC for the doubled-carrier single cycle detector is absent for the QPSK signal of interest because there is no cyclic feature at $\alpha=2 f_{c}$. 
modeled as a cyclostationary random process, reflecting the characteristic property of regenerative periodicity. Since we are concerned here with interception, the data collection parameter $T$ of the detection devices should be large compared to the correlation time (approximate width of the autocorrelation function) of the SOI or, equivalently, the reciprocal of the bandwidth of SOI. For example, for a spreadspectrum PSK SOI, the collection time $T$ should greatly exceed the chip interval $T_{c}$.

For weak signals in WGN, the maximum- likelihood (ML) signal detection criterion leads to the following approximate sufficient statistic [3]

$$
y_{m l}(t) \propto \int_{t-T / 2}^{t+T / 2} \int_{t-T / 2}^{t+T / 2} R_{s}(u, v) x(u) x^{*}(v) d u d v .
$$

It can be shown that this quadratic form is asymptotically (input $S N R \rightarrow 0$ ) optimal even the weak SOI is not Gaussian [5]. Thus, for weak-signal detection, the optimum detector implements a quadratic transformation of the received data and compares the resultant statistic to a threshold.

The actual device specified by (15) depends on the particular signal model employed. If the signal is modeled as (almost) cyclostationary, then the autocorrelation is given by (8) and the resulting device can be expressed as multicycle detector [3]

$$
y_{m c}(t)=\sum_{\alpha} \int_{-\infty}^{\infty} S_{s}^{\alpha}(f)^{*} S_{x_{T}}^{\alpha}(t, f) d f
$$

where the sum is over all $\alpha$ for which the spectral correlation function $S_{s}^{\alpha}(f)$ is not identically zero. The function $S_{x_{T}}^{\alpha}(t, f)$ is the cyclic periodogram that is defined by

$$
S_{x_{T}}^{\alpha}(t, f) \stackrel{\Delta}{=} \frac{1}{T} X_{T}(t, f+\alpha / 2) X_{T}^{*}(t, f-\alpha / 2),
$$

In equation (17), $X_{T}(t, f)$ is the Fourier transform of the segment of the received signal with length $T$ and is given by

$$
X_{T}(t, f)=\int_{t-T}^{t+T} x(u) e^{-i 2 \pi f u} d u .
$$

If the signal is modeled as stationary, then only the $\alpha=0$ term in (16) is non-zero and we obtain the optimum radiometer

$$
y_{r}(t)=\int_{-\infty}^{\infty} S_{s}^{0}(f)^{*} S_{x_{T}}^{0}(t, f) d f .
$$

Various arguments can be constructed for using the magnitude of only one $\alpha \neq 0$ term in (16)

$$
y_{s c}(t)=\int_{-\infty}^{\infty} S_{s}^{\alpha}(f)^{*} S_{x_{T}}^{\alpha}(t, f) d f \quad ; \alpha=0 .
$$

The device that compares the magnitude of the statistic $(20)$ to a threshold is referred to as a single-cycle detector (or just cycle detector). By way of interception, the cycle 
As shown in Figs. 2-3, the performance of the doubled-carrier single cycle detector is far superior to both the bit-rate single cycle detector and the radiometer. The bit-rate single cycle detector, however, is better than the radiometer. The case that is illustrated at Fig. 2 is for a collect of 16 symbols of signal of interest whereas the case that is illustrated at Fig. 3 is for a collect of 32 symbols of signal of interest. Comparison of these Figures indicates that increasing the collect time of signal of interest enhances the ROC.

For example, in Fig. 4 , for a probability of false alarm of $P_{F A}=0.02$, the radiometer has a probability of detection of $P_{D 1}=0.30$, and the bit-rate single cycle detector has a probability of detection of $P_{D 2}=0.70$. In Fig. 5 , the previous values of probability of detection become as follows, $P_{D 1}=0.50$, and $P_{D 2}=0.85$. All previous discussed cases are at $S N R=-5 \mathrm{~dB}$. To illustrate the effect of the value of signal to noise ratio on the performance of ROC's of the considered detectors, the aforementioned cases are discussed at another value of signal to noise ratio, SNR=$10 \mathrm{~dB}$. As shown at Figs. $2-5$ and at Figs. 6-9, the performance of ROC's at SNR=-5 $\mathrm{dB}$ is better than that case of $\mathrm{SNR}=-10 \mathrm{~dB}$.

The previous discussion and its related figures show advantages of cyclic feature detectors on the radiometer. These cyclic feature detectors provide an improvement of receiver operating characteristics even at low values of signal to noise ratio. Thus, weak signals may be intercepted using the property of the cyclostationarity.

\section{5- CONCLUSIONS}

This paper is concerned with the receiver operating characteristics (ROC) for different types of detectors. Receiver operating characteristics are improved depending on cyclic features of the intercepted signal. For the purposes of signal interception, the signal of interest is appropriately modeled as a cyclostationary random process. Cyclostationarity of an intercepted signal is characterized by the spectral correlation function. In this paper a new method for fast computation of the spectral correlation function is provided. The receiver operating characteristic is evaluated. Three types of detectors are used. These detectors are the radiometer, the bit-rate single cycle detector, and doubled-carrier single cycle detector. The ROC of these three detectors is quantitively evaluated through computer simulation. It is found that, the performance of the doubled-carrier single cycle detector is far superior to both the bit-rate single cycle detector and the radiometer. The bit-rate single cycle detector, however, is better than the radiometer. For example, at SNR of $-5 \mathrm{~dB}$, for a probability of false alarm of $2 \%$, the radiometer has a probability of detection of $40 \%$, the bit-rate single cycle detector has a probability of detection of $70 \%$, and the doubled-carrier single cycle detector has a probability of detection of $93 \%$. This improvement is referred to the reduction of noise by the cyclic detectors.

\section{REFERENCES}

[1] W. A. Gardner," Signal interception: A unifying theoretical framework for feature detection," IEEE Trans. Commun. Vol. 36, pp. 897-906, Aug. 1988.

[2] W. A. Gardner, Introduction to Random Processes with Applications to 
Signals and Systems, 2nd ed New York; McGraw-Hall, 1989.

[3] W. G. Gardner, "Signal interception: Performance advantages of cyclicfeature detectors," IEEE Trans. Comm., vol. 40, pp. 149-159, Jan. 1992.

[4] R. A. Boyles and W. A. Gardner," Cycloergodic properties of discreteparameters non-stationary stochastic processes," IEEE Trans. Inform. Theory, vol. IT-29, pp. 105-114, Jan. 1983.

[5] W. A. Gardner," Structural characterization of locally optimum detectors in terms of locally optimum estimators and correlators," IEEE Trans. Inform. Theory, vol. IT-28, pp. 924-932, Nov. 1982.

[6] A. Al-Makhlouf, " Signal Interception Using Advanced Processing Techniques," Phd. thesis, MTC, Cairo, Dec. 2000.
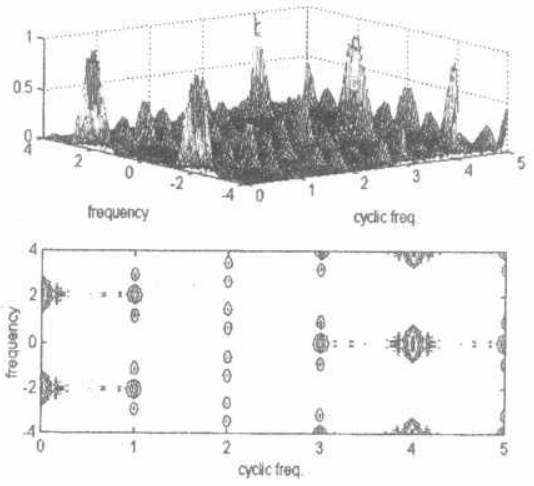

(a)
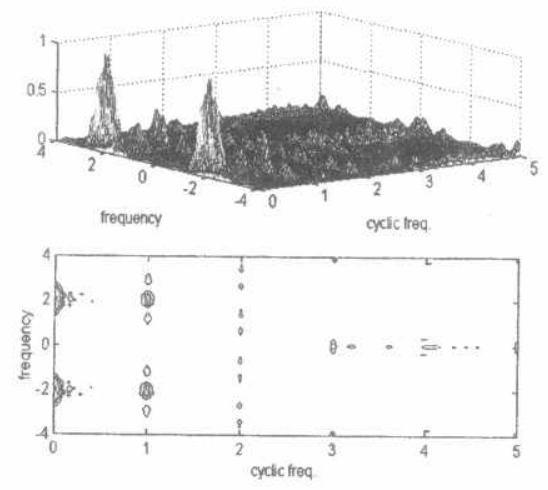

(b)

Fig.1: a) SCF of BPSK signal using F-D method b) SCF of QPSK signal using F-D method
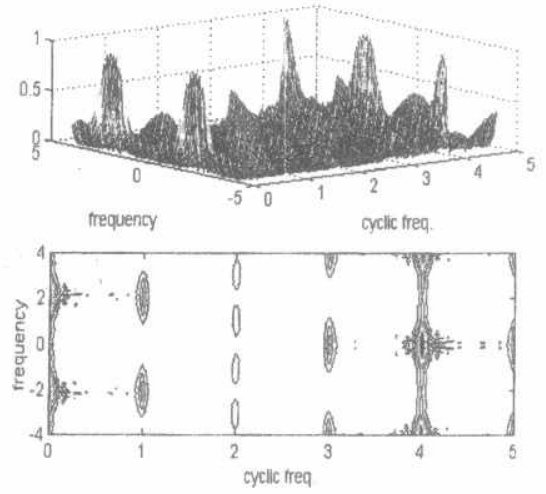

(c)
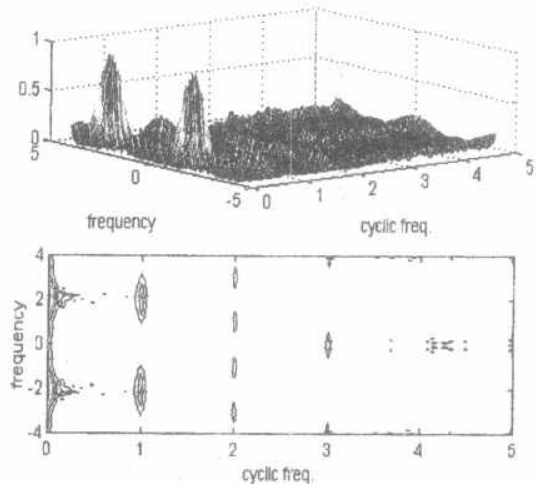

(d)

c) SCF of BPSK signal using T-D method d) SCF of QPSK signal using T-D method 


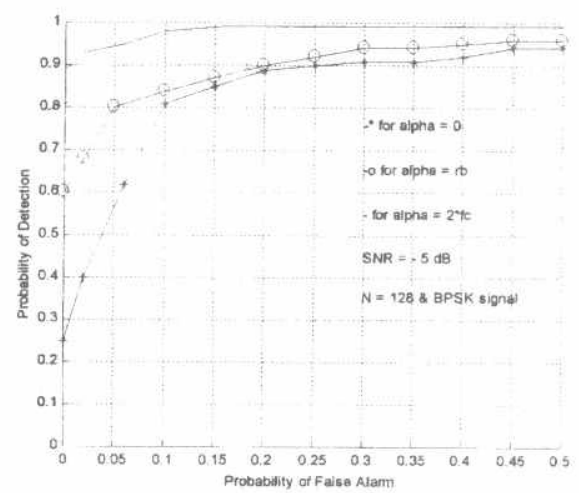

Fig. 2: ROC of a BPSK signal at $\mathrm{SNR}=-5 \mathrm{~dB}$ and $\mathrm{N}=128$ samples.

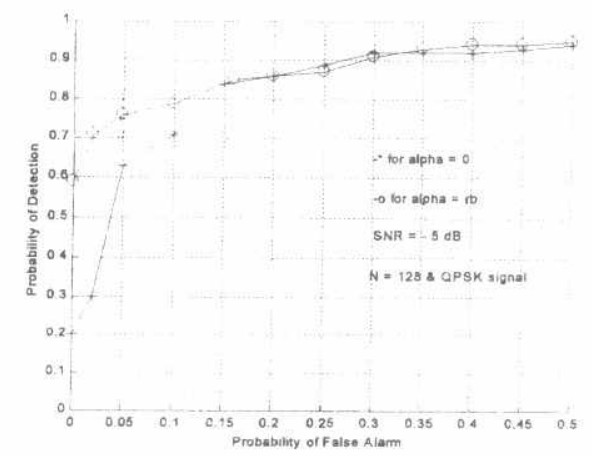

Fig. 4: ROC of a QPSK signal at SNR $=-5 \mathrm{~dB}$ and $\mathrm{N}=128$ samples.

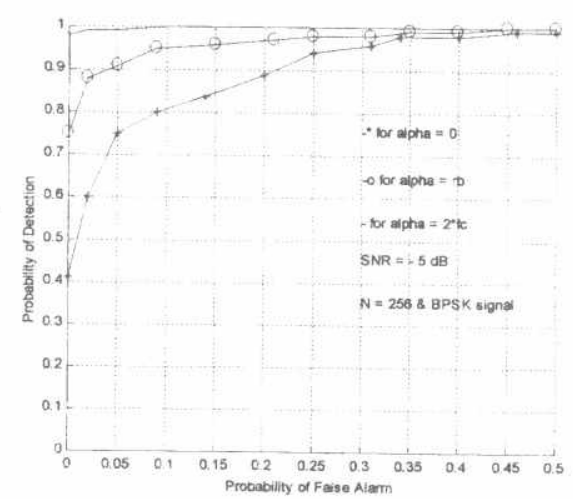

Fig. 3: ROC of a BPSK signal at $\mathrm{SNR}=-5 \mathrm{~dB}$ and $\mathrm{N}=256$ samples.

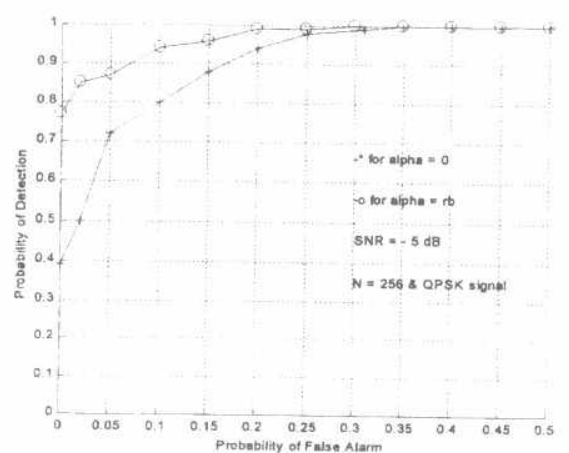

Fig. 5: ROC of a QPSK signal at $\mathrm{SNR}=-5 \mathrm{~dB}$ and $\mathrm{N}=256$ samples. 


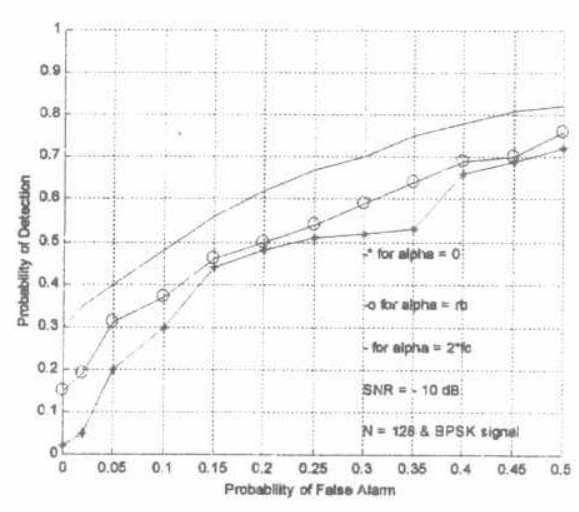

Fig. 6: ROC of a BPSK signal at $\mathrm{SNR}=-10 \mathrm{~dB}$ and $\mathrm{N}=128$ samples

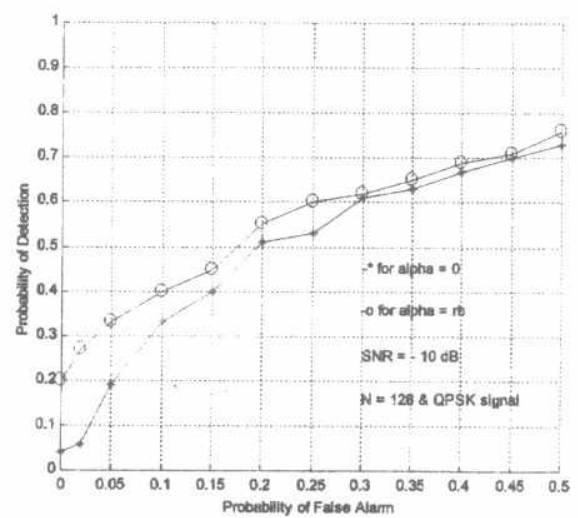

Fig. 8: ROC of a QPSK signal at $\mathrm{SNR}=-10 \mathrm{~dB}$ and $\mathrm{N}=128$ samples.

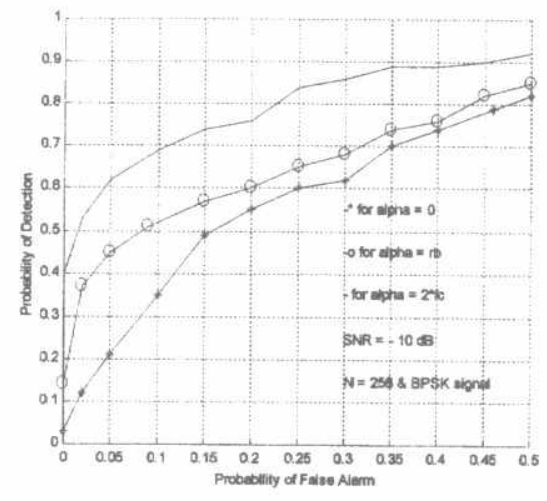

Fig. 7: ROC of a BPSK signal at $\mathrm{SNR}=-10 \mathrm{~dB}$ and $\mathrm{N}=256$ samples.

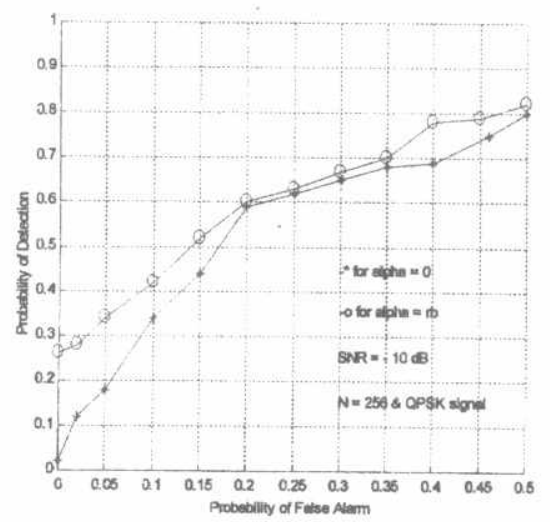

Fig. 9: $R O C$ of a BPSK signal at $S N R=-10 \mathrm{~dB}$ and $\mathrm{N}=256$ samples. 